\title{
Short- and intermediate-time behavior of the linear stress relaxation in semiflexible polymers
}

\author{
P. Dimitrakopoulos, J. F. Brady, and Z.-G. Wang \\ Division of Chemistry and Chemical Engineering, California Institute of Technology, Pasadena, California 91125
}

(Received 19 April 2001; published 26 October 2001)

\begin{abstract}
The linear viscoelasticity of semiflexible polymers is studied through Brownian Dynamics simulations covering a broad range of chain stiffness and time scales. Our results agree with existing theoretical predictions in the flexible and stiff limits; however, we find that over a wide intermediate-time window spanning several decades, the stress relaxation is described by a single power law $t^{-\alpha}$, with the exponent $\alpha$ apparently varying continuously from $1 / 2$ for flexible chains, to $5 / 4$ for stiff ones. Our study identifies the limits of validity of the $t^{-3 / 4}$ power law at short times predicted by recent theories. An additional regime is identified, the "ultrastiff" chains, where this behavior disappears. In the absence of Brownian motion, the purely mechanical stress relaxation produces a $t^{-3 / 4}$ power law for both short and intermediate times.
\end{abstract}

DOI: 10.1103/PhysRevE.64.050803

PACS number(s): 61.41.+e, 83.60.Bc, 83.10.Mj, 87.15.- -v

The present study identifies the effects of local stiffness on the short- and intermediate-time relaxation of semiflexible polymers. Common examples of these polymers include biopolymers such as DNA, actin filaments, microtubules and rodlike viruses, as well as a host of stiff synthetic polymers such as Kevlar and polyesters. Although we explicitly consider the relaxation of a single polymer chain in a viscous solvent, our results should be valid even for concentrated polymer solutions and networks as long as the relaxation of interest occurs on length scales shorter than that characterizing the entanglements or crosslinks. We focus on the scaling behavior in the stress relaxation based on the relation between the polymer configuration and stress, covering a wide range of time scales and flexibility (i.e., from flexible to very stiff chains). Our study identifies the limits of validity of the power law decay of $t^{-3 / 4}$ at short times predicted in recent theoretical studies $[1,2]$. In addition, we identify the effects of the anomalous diffusion associated with the longitudinal relaxation at intermediate times $[3,4]$. Over a broad range of intermediate times, our results show that the stress relaxation is described by a single power law decay $t^{-\alpha}$, with the exponent $\alpha$ apparently varying continuously from $1 / 2$ for flexible chains, to $5 / 4$ for stiff ones, the latter confirming a recent theoretical prediction [5]. We identify an additional regime of rigidity, the "ultrastiff" regime, where the entire shortand intermediate-time relaxation shows only a $t^{-5 / 4}$ decay. Finally, we suppress the Brownian motion to identify the influence of purely mechanical relaxation.

A discretized version of the wormlike chain model $[6,7]$ is employed based on a Brownian Dynamics method developed in Ref. [8]. This method considers a (flexible) bead-rod model with fixed bond lengths and ignores hydrodynamic interactions among beads as well as excluded-volume effects. For stiff chains, hydrodynamics has little effect on intrachain dynamics, and in concentrated solutions and melts, the hydrodynamic interactions are screened [6]. The polymer chain is modeled as $(N+1)$ identical beads connected by $N$ massless links of fixed length $b$. The position of bead $i$ is denoted as $\boldsymbol{X}_{i}$, while the link vectors are given by $\boldsymbol{d}_{i}$ $=\boldsymbol{X}_{i+1}-\boldsymbol{X}_{i}$. To account for polymer stiffness, a bending energy proportional to the square of the local curvature is added. For a continuous chain $\phi^{\text {bend }}=(1 / 2) \mathcal{E} b \int_{0}^{L}(\partial \hat{\boldsymbol{d}} /$ $\partial s)^{2} \mathrm{~d} s$, where $L$ is the (constant) contour length of the chain for polymer solutions or the entanglement length for networks, and $\hat{\boldsymbol{d}}$ the local unit tangent. The bending energy $\mathcal{E}$ is related to the persistence length $L_{p}$ via $\mathcal{E} / k_{B} T \equiv L_{p} / b$, where $k_{B}$ is the Boltzmann constant. The bending energy of the discrete model is given by $\phi^{\text {bend }}=\mathcal{E} \Sigma_{i=1}^{N-1}\left(1-\boldsymbol{d}_{i} \cdot \boldsymbol{d}_{i+1} / b^{2}\right)$. For a fixed $b$, the properties of the polymer chain are specified by the number of links $N$ and the dimensionless bending energy $E=\mathcal{E} / k_{B} T$.

Assuming that the bead inertia is negligible, the sum of all forces acting on each bead $i$ must vanish, which leads to the following Langevin equation

$$
\zeta \frac{\mathrm{d} \boldsymbol{X}_{i}}{\mathrm{~d} t}=\boldsymbol{F}_{i}^{\text {bend }}+\boldsymbol{F}_{i}^{r \text { and }}+\boldsymbol{F}_{i}^{\text {tension }}+\boldsymbol{F}_{i}^{\text {cor }}
$$

where the friction coefficient $\zeta$ is assumed to be uniform [8]. The bending force $\boldsymbol{F}_{i}^{b e n d}$ is derived from the chain bending energy, and $\boldsymbol{F}_{i}^{\text {rand }}$ is the Brownian force due to the constant bombardments of the solvent molecules. The force $\boldsymbol{F}_{i}^{\text {tension }}$ $=T_{i} \boldsymbol{d}_{i}-T_{i-1} \boldsymbol{d}_{i-1}$, where $T_{i}$ is a constraining tension along the direction of each link $\boldsymbol{d}_{i}$, ensures the link inextensibility. Finally, $\boldsymbol{F}_{i}^{c o r}$ is a corrective potential force added so that the equilibrium probability distribution of the chain configurations is Boltzmann $[8,9]$. The resulting system of equations may be solved in $O(N)$ operations as described in Ref. [8]. Ensemble averages are accomplished by generating independent initial equilibrium configurations using a Monte Carlo Metropolis algorithm based on the chain bending energy.

The polymer stress is calculated from $\boldsymbol{\sigma}=-\sum_{i=0}^{N}\left(\boldsymbol{X}_{i}\right.$ $\left.-\boldsymbol{X}_{c}\right) \boldsymbol{F}_{i}^{\text {total }}$, where $\boldsymbol{F}_{i}^{\text {total }}$ is the sum of all forces appearing on the right-hand side of Eq. (1), and $\boldsymbol{X}_{c}$ the center of mass of the chain. Based on the fluctuation-dissipation theorem, the linear stress relaxation is described by the stress autocorrelation function at equilibrium, $C_{\text {stress, } i j}(t)$ $\equiv\left\langle\sigma_{i j}(t) \sigma_{i j}(0)\right\rangle$ with $i, j=1,2,3$. At equilibrium there is no preferred direction and we calculate the arithmetic mean of the three diagonal components, $C_{n o r m}$, and of the remaining 
six components, $C_{\text {shear }}$, of the correlation tensor, $\boldsymbol{C}_{\text {stress }}$. Both functions show similar relaxation and thus we present only $C_{\text {shear }}$.

The Brownian and bending forces give rise to two microscopic time scales. The Brownian time scale is associated with the diffusive motion of one bead and is given by $\tau_{\text {rand }}=\zeta b^{2} / k_{B} T$. The bending time scale is associated with the relaxation of the angle between two successive links. For large bending energy $E \gg 1$, this time scale is given by $\tau_{\text {bend }}=\zeta b^{2} / \mathcal{E}=\tau_{\text {rand }} / E \ll \tau_{\text {rand }}$. Although this time scale does not manifest itself explicitly in the continuum representation of a chain, a cutoff is required for any continuum model to be well-defined. Therefore, this time scale represents the smallest possible time scale in the current problem.

At times $t \sim \tau_{\text {bend }}$, only the chain ends show significant transverse relaxation; the angles in the chain interior have not yet relaxed due to the constraints imposed by the presence of neighbor beads. At later times, these angles, as well as portions of the entire chain, show significant transverse relaxation [10]. The end of the transverse fluctuations (as well as the end of the intermediate-time behavior) occurs when the entire polymer chain shows significant transverse relaxation. This time represents the relaxation of the longest bending mode and is given by $\tau_{\perp}=\zeta L^{4} /\left(b^{2} \mathcal{E}\right)=\tau_{\text {rand }} N^{4} / E$, readily obtained from the linearization of Eq. (1) around the straight rod limit $[1,2,4]$. For a long chain $N \gg 1, \tau_{\perp}$ $\gg \tau_{\text {bend }}$; thus these two time scales are well separated.

Due to the conservation of the polymer contour length, the transverse fluctuations result in longitudinal fluctuations. As discussed in Refs. [3,4], the longitudinal tension propagates (as anomalous diffusion) a distance $l_{\|}(t)$ $\sim t^{1 / 8} E^{5 / 8}\left(k_{B} T / \zeta\right)^{1 / 8} b^{3 / 4}$ along the polymer backbone during time $t$. At short times this propagation cannot cover the entire contour length, and the stress relaxation is dominated by transverse fluctuations. At intermediate times when the longitudinal tension influences the entire chain, both types of fluctuations affect the stress relaxation. This change occurs when $l_{\|} \sim L$, giving a time scale $\tau_{\|}=\zeta L^{8} /\left(k_{B} T b L_{p}^{5}\right)$ $=\tau_{\text {rand }} N^{8} / E^{5}[5]$. Therefore, at short times $\tau_{\text {bend }} \leqslant t \ll \tau_{\|}$, stress relaxation is caused by transverse relaxation only. At intermediate times $\tau_{\|} \leqslant t \leqslant \tau_{\perp}$, both transverse and longitudinal relaxations contribute to the polymer stress decay. We emphasize that the expressions for $\tau_{\perp}$, and $\tau_{\|}$, as well as the related discussion, are valid only for stiff chains $E \geqslant N$.

Figure 1(a) shows the shear stress autocorrelation $C_{\text {shear }}$ at early times, for different values of $E$ and $N$. The initial decay from the plateau is caused by transverse fluctuations at the chain ends at times $t \sim \tau_{\text {bend }}$. This interpretation is corroborated by Fig. 1(b), which shows the relaxation of the angle at the chain ends, $C_{\text {angle }} \equiv\langle x(t) x(0)\rangle-\langle x\rangle^{2}$, with $x(t)$ the transverse fluctuation at the chain ends, $x$ $=\sqrt{b^{2}-\boldsymbol{d}_{i} \cdot \boldsymbol{d}_{i+1}}(i=1$ or $N-1)$. Therefore, the initial stress decay depends only on the bending energy $E$; away from the plateau the stress relaxation depends on $N$ as well, due to the influence of the transverse relaxation of the chain interior. Based on the correspondence between the conformation and stress relaxation, the bending time scale $\tau_{\text {bend }}$ for any bending energy $E$ can be identified as $\tau_{\text {bend }} \equiv 2\langle x x\rangle / D_{1}$, where
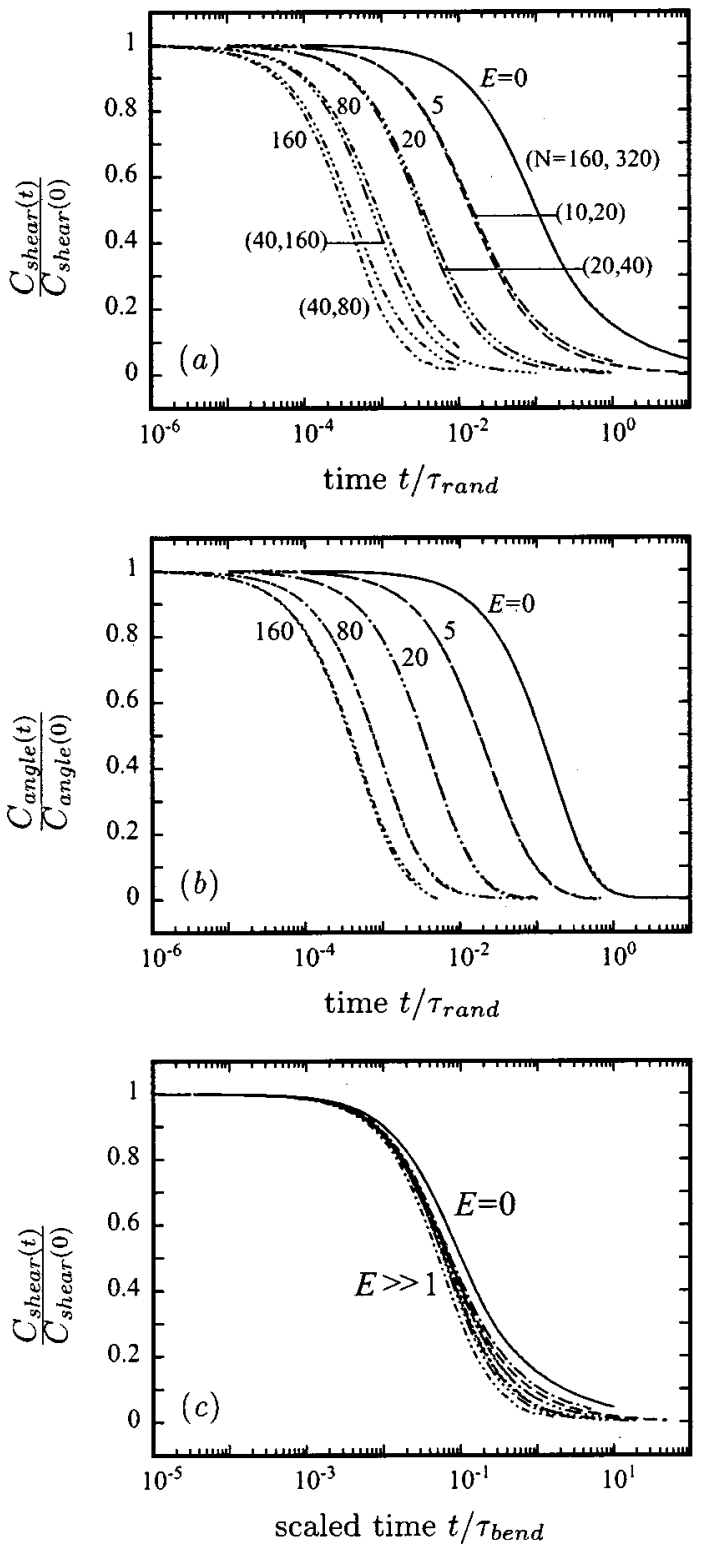

FIG. 1. Relaxation of the polymer chain at early times. (a) Shear stress autocorrelation $C_{\text {shear }}$ vs time $t / \tau_{\text {rand }}$ for several values of $E$. For each value of $E$ two different values of $N$ are included. (b) Angle autocorrelation $C_{\text {angle }}$ vs time $t / \tau_{\text {rand }}$ for the same values of $E$ and $N$ as in (a). (c) Shear stress autocorrelation $C_{\text {shear }}$ vs time $t$ scaled by the microscopic bending time scale $\tau_{\text {bend }}$, for the same values of $E$ and $N$ as in (a), showing the good scaling behavior for the initial decay from the plateau.

$D_{1}$ is the diffusivity of a single bead $k_{B} T / \zeta$. Further analysis reveals that $\tau_{\text {bend }}=\tau_{\text {rand }}\left[1-(1+2 E) \mathrm{e}^{-2 E}\right] /\left[E\left(1-\mathrm{e}^{-2 E}\right)\right]$ [10]. For large bending energy $E \gg 1$, this time scale is $\tau_{\text {bend }}=\tau_{\text {rand }} / E \ll \tau_{\text {rand }}$, while it approaches smoothly the Brownian time scale $\tau_{\text {rand }}$ as $E \rightarrow 0$. Good scaling behavior for the initial decay from the plateau is obtained when the shear stress correlation $C_{\text {shear }}$ is plotted versus the scaled time $t / \tau_{\text {bend }}$ in Fig. 1(c). We note that this microscopic time scale was not considered in previous studies employing the continuous wormlike chain model $[1,2,4,5]$. 

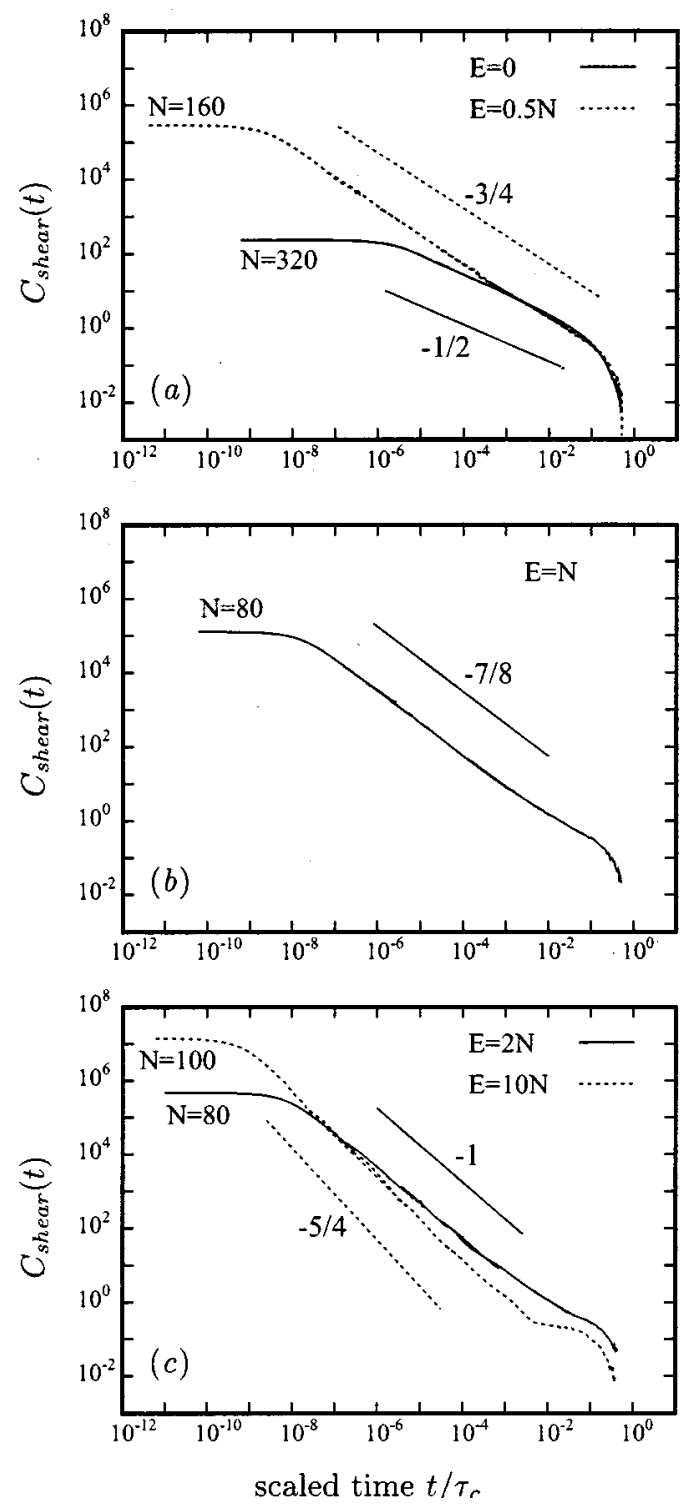

FIG. 2. Shear stress autocorrelation $C_{\text {shear }}$ [in units of $\left(k_{B} T\right)^{2}$ ] vs time $t$ scaled by the translational diffusion time $\tau_{c}$ at short times.

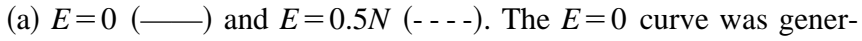
ated using chains with $N=10,20,40,160,320$, while the $E=0.5 N$ curve with $N=10,20,40,80,160$. (b) $E=N$ (—) generated with $N=10,20,40,60,80$. (c) $E=2 N$ ( - ) and $E=10 N(---)$. The $E$ $=2 N$ curve was generated with $N=5,10,20,40,80$, while the $E$ $=10 \mathrm{~N}$ curve with $N=5,10,20,40,100$. The fractional exponents are merely to illustrate the changing slope and not meant to imply a precise numerical value.

We now focus on the polymer stress at times $\tau_{\text {bend }} \leqslant t$ $\leqslant \tau_{\perp}$. In Fig. 2 we plot the shear stress correlation function $C_{\text {shear }}$ versus time $t$ for specific values of the chain stiffness relatively to the chain contour length, i.e., for $E / N$ $=0,0.5,1,2,10$. The time has been scaled by the translational diffusion time $\tau_{c}$, which describes the long-time behavior of the polymer stress. This behavior is associated with the diffusive translation or rotation of the entire chain as discussed in Ref. [10]. The time scale $\tau_{c}$ is defined as the ratio of a mean square length of the entire chain (i.e., the radius of gyration $R_{g}$ ) to the diffusivity $D_{C}$ of the chain's center of mass; $\tau_{c} \equiv\left\langle R_{g}^{2}\right\rangle / D_{C}$ with $D_{C}=k_{B} T /(N+1) \zeta \quad$ [10]. Each curve is generated by first performing runs using several different $N$ at a given value of $E / N$ for a time window spanning 5-7 decades, and then scaling the time with $\tau_{c}$. The fact that the curves for different $N$ collapse to a single curve at a given $E / N$ is strong indication of dynamic scaling.

The results in Fig. 2 reveal that for a given chain, after the plateau at early times, the stress relaxes in a power law manner towards the long-time regime. For a wide range of chain stiffness, this stress decay appears to follow a single power law, which varies continuously from $t^{-1 / 2}$ for completely flexible chains, to $t^{-5 / 4}$ for stiff ones. This behavior is in contrast to the long-time behavior, where two distinct regimes $(E \ll N, E \geqslant N)$ were found with respect to the polymer stiffness [10]. The two limiting values of the power law are in agreement with the analytical predictions of the Rouse model and the recent work of Ref. [5], respectively. However, our results cover a much broader range of chain stiffness than these limiting cases.

The intermediate time behavior terminates at times $t$ $\sim \tau_{\perp}$, while the long-time behavior becomes dominant at times $\tau_{c}$. The gap between $\tau_{\perp}$ and $\tau_{c}$ broadens for $E \gg N$; in this case $\tau_{c} \sim \tau_{\text {rigid }} \sim N^{3}[6,10]$ and $\tau_{\perp} / \tau_{c} \sim N / E$. This is responsible for the appearance of a second plateau at times $t$ $\sim \tau_{\perp}$ for stiff chains, as shown in Fig. 2(c) (see the end of the $E=10 N$ curve, for times $t / \tau_{c} \approx 5 \times 10^{-3}-10^{-1}$ ).

In spite of the fact that we employed rather long chains, we were unable to verify the existence of the $t^{-3 / 4}$ law for finite bending energy at short times, $\tau_{\text {bend }} \leqslant t \ll \tau_{\|}[1,2]$. From our analysis of the time scales, it is clear that for $\tau_{\|}$to constitute a distinct time scale, we need $N \ll E \ll N^{2}$. The left half of this inequality, which reflects the separation of $\tau_{\|}$and $\tau_{\perp}$, can be easily satisfied for moderately large values of $N$ and $E$. On the other hand, the right half of the inequality, which indicates the separation of $\tau_{\text {bend }}$ and $\tau_{\|}$, would require simulations using very long chains that would be computationally very expensive. Therefore, our results in Fig. 2 show the stress relaxation for $\tau_{\text {bend }} \sim \tau_{\|} \leqslant t \leqslant \tau_{\perp}$, where both transverse and longitudinal fluctuations contribute to the stress decay.

The short-time $t^{-3 / 4}$ decay, predicted by recent theoretical studies [1,2], should disappear for chains with $E \gg N^{2}$. In this limit the longitudinal time scale $\tau_{\|}$becomes smaller than $\tau_{\text {bend }}$, the smallest time scale present. A logical conclusion is that $\tau_{\|}$and the associated predictions [1,2] become irrelevant in this regime, which we call ultrastiff. In this regime, the entire short- and intermediate-time relaxation should exhibit only a $t^{-5 / 4}$ decay. Note that the transverse time scale $\tau_{\perp}=\zeta L^{4} /\left(b^{2} \mathcal{E}\right)=\tau_{\text {rand }} N^{4} / E$ can still be longer than the Brownian time scale $\tau_{\text {rand }}$ associated with the diffusive motion of a single bead. For $E \gg N^{4}$, the bending relaxation of the entire chain occurs faster than the Brownian motion of a single bead, and this condition defines the rigid regime, where on times longer than $\tau_{\text {rand }}$, only rotational relaxation is observable.

Based on the above analysis, we can classify the dynamic behavior of long wormlike chains into the following re- 
gimes: completely flexible $(E=0)$, flexible $(E \ll N)$, semistiff $(E \approx N)$, stiff $\left(N^{2} \gg E>N\right)$, ultrastiff $\left(N^{4} \gg E \gg N^{2}\right)$, and rigid $\left(E \gg N^{4}\right)$. We comment that similar analysis can be applied to a segment of the chain in determining its local dynamic behavior, and the different regimes can be accessed through a change of $N$ for a given polymer.

Our results are in good agreement with current experimental findings. It is well-known that flexible polymers show high-frequency $\omega^{1 / 2}$ scaling of the shear modulus [6], in agreement with our $E=0$ results. Concentrated solutions and networks of actin filaments were found to exhibit a highfrequency viscoelastic modulus $\omega^{0.75-0.78}[11-13]$. In addition, our results may explain recent experimental findings that suggest a high-frequency viscoelastic modulus varying from $\omega^{3 / 4}$ to $\omega^{1}[13,14]$. Finally, we hope that our study motivates experiments that cover all the stiffness regimes that we have identified.

To understand the effects of mechanical bending, we performed purely deterministic simulations by suppressing the Brownian motion. We start from configurations having small enough perturbations (so as to be in the linear regime) to the initially straight chains. The initial perturbations are generated using a Monte Carlo Metropolis algorithm at chosen initial temperatures. The temperature is then suddenly dropped to $T=0$ and the chain is allowed to relax towards the equilibrium straight configuration. Figure 3 shows the relaxation of the polymer normal stress, $\sigma_{\text {norm }}(t)$, defined as the arithmetic mean of the three diagonal components of the stress tensor. The figure shows the existence of a $t^{-3 / 4}$ power law. In this case, the relaxation is governed by transverse motion and the power-law behavior is bracketed by the time scales $\tau_{\text {bend }} \equiv \zeta b^{2} / \mathcal{E}$ and $\tau_{\perp} \equiv N^{4} \tau_{\text {bend }}$, i.e., it is valid for

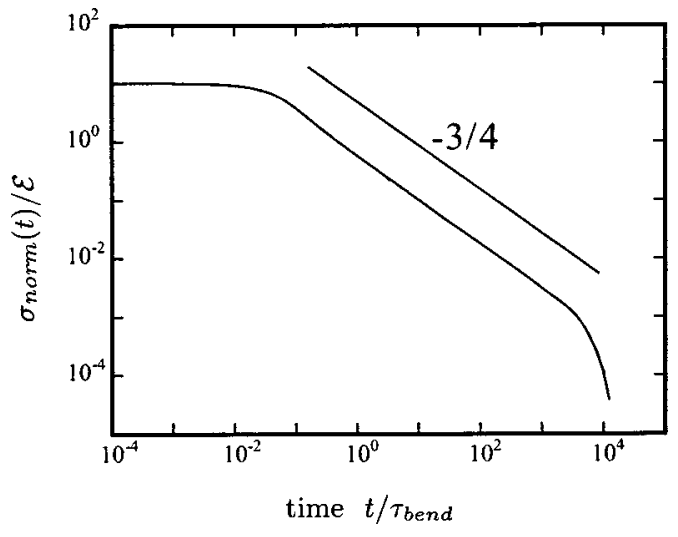

FIG. 3. Deterministic relaxation of the polymer stress for a chain with $N=40$. The initial state $(t=0)$ corresponds to $T=2.5$ $\times 10^{-3} \mathcal{E} / k_{B}$.

both short and intermediate times. Recall that the short-time $t^{-3 / 4}$ relaxation is also due to transverse fluctuations. In contrast, for ultrastiff chains over the same time interval a different power law $\left(t^{-5 / 4}\right)$ was observed; this points to the essential role of thermal motion at any finite temperature.

This work was supported by the Grant No. DMR9970589 from the National Science Foundation. The computations were performed on multiprocessor computers provided by the National Center for Supercomputing Applications at Urbana, and the Center for Advanced Computing Research at Caltech. The authors wish to thank David Morse for providing access to his recent work [5] and for helpful discussions.
[1] D.C. Morse, Phys. Rev. E 58, R1237 (1998).

[2] F. Gittes and F.C. MacKintosh, Phys. Rev. E 58, R1241 (1998).

[3] D.C. Morse Macromolecules 31, 7044 (1998).

[4] R. Everaers, F. Julicher, A. Ajdari, and A.C. Maggs, Phys. Rev. Lett. 82, 3717 (1999).

[5] M. Pasquali, V. Shankar, and D. C. Morse Phys. Rev. E 64, 020802 (2001).

[6] M. Doi and S. F. Edwards, The Theory of Polymer Dynamics (Clarendon, Oxford, 1986).

[7] H. Yamakawa, Helical Wormlike Chains in Polymer Solutions
(Springer, Berlin, 1997).

[8] P.S. Grassia and E.J. Hinch, J. Fluid Mech. 308, 255 (1996).

[9] M. Fixman, J. Chem. Phys. 69, 1527 (1978).

[10] P. Dimitrakopoulos, J. F. Brady, and Z.-G. Wang (unpublished).

[11] F. Gittes et al., Phys. Rev. Lett. 79, 3286 (1997).

[12] J. Xu, A. Palmer, and D. Wirtz, Macromolecules 31, 6486 (1998).

[13] T. Gisler and D.A. Weitz, Phys. Rev. Lett. 82, 1606 (1999).

[14] J. H. van Zanten (unpublished). 